\title{
BER analysis in different channel with variant channel capacity
}

\author{
Mukesh Bhaskar* and Anil Khandelwal \\ Department of Electronics and Communication, VNS Group of Institutions, Bhopal, India
}

\section{(C2017 ACCENTS}

\begin{abstract}
This paper addresses and highlights the drawback of communication systems which is mainly suffer from signal attenuation and spectrum fading and it is because of the diverse mobility factor associated with it. The orthogonal frequency division multiplexing (OFDM) is determined as a multicarrier system that is able to achieve high efficiency in terms of spectral efficiency. Multiple input multiple output (MIMO) arrangement gives upgraded limit the same transmits power. The combinations of OFDM and MIMO have the ability to increase diversity gain and the capacity of the system in time variant and frequency variant channels. MIMO-OFDM arrangement is found to perform better against multi-way fading and the differing channel conditions, than the previous methods. Precoding is another strategy that can be connected which enhances the execution of a MIMO OFDM framework. In this dissertation, the BER performance has been analyzed of a MIMO-OFDM framework utilizing precoding is recreated for Quadrature amplitude modulation (QAM). 16-QAM to 128-QAM has been considered for regulation configurations. The main aim of this dissertation is to demonstrate the data with the considerable change in temperature.
\end{abstract}

\section{Keywords}

Temperature and BER, Quadrature amplitude modulation, AWGN, Rayleigh fading channel, OFDM and MIMO.

\section{Introduction}

OFDM is a frequency division multiplexing (FDM) plan utilized as a progressed multi-carrier change method [1,2] in a manner of speaking OFDM is repeat division multiplexing of multi-bearers which are orthogonal to each other i.e. they are put accurately at the nulls in the regulation spectra of each other. This makes OFDM appallingly more compelling [3]. In OFDM data is isolated into a couple parallel data streams or sub-channels, one for each sub conveyor which are orthogonal to each other notwithstanding the way that they cover appallingly Each sub-transporter is controlled with a normal regulation plan, (for example, QAM or PSK) at a low picture rate keeping up total data rates like conventional single-transporter change plots in the same transmission limit. In today's circumstance MIMO is to a great degree accommodating with the mix of OFDM structure. Abusing the flexibility of MIMO systems remembering the final objective to have high data rates is an especially charming investigation topic for future booking arrangement arranges and their applications. Distinctive data different yield (MIMO) systems offer much greater channel limit over standard single-data single-yield structure.

\footnotetext{
*Author for correspondence
}

As of various transmit Algorithms have been delivered to experience as far as possible in the MIMO systems [4, 5].Furthermore, in MIMO structures, resulting to selecting the social event of customers with the at present most prominent achievable rates managed by a package scheduler in without fail opening, we need to delegate them to the transmitter's radio wires in such a course, to the point that we can finish the best throughput in the system. Grouped qualities strategies, for instance, space-time coding have gotten a ton of thought in view of their ability to give higher unearthly profitability than routine single-data single-yield frameworks [6-9]. While applying this methodology in a repeat specific channel, a space-time equalizer is required at the recipient to compensate for the impedance [10].

This multipath spread reasons optional time dispersing, tightening, and stage development, known as obscuring, in the got signal $[11,12]$. Obscuring is achieved by deterrent between two or more types of the transmitted sign which connected at the authority at to some degree unmistakable times [13]. DSCDMA system has the upsides of extending as far as possible nearby the resistance against staying [1416]. In multi-customer CDMA structures, various access interface (MAI) is seen as one of the standard wellsprings of execution debasement. Flexible 
isolating techniques have been adequately used to level the direct and in this way lessen the MAI in the DS-CDMA structure [17, 18].

\section{Related work}

In 2011, Dubey et al. [19] proposed a Modernize SLM (MSLM) plan to lessen the PAPR by using the psyche boggling sign separate into certifiable and whimsical parts and independently arrange progression various honest to goodness and nonexistent bit of complex banner then select slightest PAPR indication of real and whimsical and these are solidify. The proliferation show fulfills incredible PAPR, which is an in number contender for Future remote correspondence.

In 2011, Ropokis et al. [20] present a symptomatic piece slip rate (BER) execution examination of three perceive and-forward action under a run of the mill framework. More especially, as far as possible and association adaptable arrangements are focused on, which differentiate in the way the decision on the transmitted picture is sent from the hand-off to the destination. The examination is finished for a single hand-off DaF transmission tradition and considers the picture decision botches that may happen at the exchange. Clear close structure demonstrative BER expressions are deduced for each one of the three arrangements, which are completely affirmed by entertainments.

In 2012, Samir et al. [21] propose a change to the execution of a Direct Sequence Code Division Multiple Access (DS-CDMA) system by utilizing an adaptable channel as a part of the region of unmistakable staying routines. To fight the impact of such staying, the flexible channel utilizes three adaptable estimations which are the Variable StepSize Affine Projection (VSS-APA) count, the Generalized Normalized Gradient Descent (GNGD) figuring, and the Generalized Square-ErrorRegularized (GSER) NLMS computation. According to the inventors these counts have the advantages of snappy meeting, low predictable state mean squared mix-up and the ability to upgrade the bit mix-up rate (BER) execution of the standard CDMA structure, in the region of multi-way, various passageway, and unmistakable staying signs. Results exhibit that the VSS-APA defeats distinctive estimations in the region of surge staying. While in the region of midway band staying the GSER-NLMS flexible channel gives the best execution.
In 2014, Le et al. [22] show a precise piece mistake rate estimation strategy for QPSK CO-OFDM transmission taking into account the likelihood thickness capacity of the got QPSK images. Creators had been thought about some known methodologies, including information supported and non-information helped blunder vector size, they demonstrate that the proposed technique offers the most exact appraisal of the framework execution for both single channel and wavelength division multiplexing QPSK CO-OFDM transmission frameworks.

In 2014, Zahed et al. [23] exhibited a diagnostic way to deal with decide the effect of recurrence counterbalance, timing jitter and added substance white Gaussian clamor (AWGN) on the bit error rate (BER) execution of a multi-transporter directsuccession code division numerous entrance (MCDS-CDMA) framework over a Rayleigh Fading Channel. The examination built up the pdf (likelihood thickness capacity) at the collector considering joined impact of blurring, timing jitter and Doppler recurrence counterbalance and so on with maximal proportion consolidating (MRC) plan. The expression for the restrictive BER adapted on a given timing blunder and blurring is determined and the normal BER is assessed in the vicinity of Multiple Access Interference (MAI) and Inter-Carrier Interference (ICI). The execution results are assessed numerically regarding SINR and BER considering framework parameters like number of clients, number of sub-transporters. The outcome demonstrates critical decay in SINR and BER execution because of blurring alongside the adjustments in parameters.

In 2015, Kumar et al. [24] proposed a wavelet based SCFDMA for investigating Bit Error Rate (BER) execution. Investigation is did utilizing distinctive wavelets and diverse adjustment plans under AWGN channel. This investigation will demonstrate that the diminishment in BER happens by utilizing wavelet change as a part of SCFDMA. Along these lines wavelet based SCFDMA gives preferable BER execution over that of DFT based SCFDMA.

In 2015, Jie et al.[25] proposed a MIMO-OFDM system, which is the combination of MIMO and OFDM technology for the high data transmission rate usability. They have suggested that MIMO-OFDM with STBC has excellent performance against Multipath effects and frequency selective fading, the BER and the coding complexity is low. Their simulation model of MIMO-OFDM system which is based on STBC is analysed on different transmission 
performances under different channels. In [26] authors has raised the same issues and depicted that it can be solved with different channel model.

\section{Proposed work}

This paper addresses and highlights the drawback of communication systems which is mainly suffer from signal attenuation and spectrum fading and it is because of the diverse mobility factor associated with it. The OFDM is determined as a multicarrier system that is able to achieve high efficiency in terms of spectral efficiency. MIMO arrangement gives upgraded limit the same transmits power. The combinations of OFDM and MIMO have the ability to increase diversity gain and the capacity of the system in time variant and frequency variant channels. MIMO-OFDM arrangement is found to perform better against multi-way fading and the differing channel conditions, than the previous methods.

Precoding is another strategy that can be connected which enhances the execution of a MIMO OFDM framework. In this dissertation, the BER performance has been analyzed of a MIMO-OFDM framework utilizing precoding is recreated for Quadrature amplitude modulation (QAM). 16-QAM to 256QAM has been considered for regulation configurations. The main aim of this dissertation is to demonstrate the data about the change of divert estimation precision in MIMO-OFDM framework. The MIMO-OFDM framework is the blend of the MIMO procedure and OFDM strategy, which is upgrading the limit, enhance the connection unwavering quality high information rate transmission for future broadband remote correspondence furthermore use for maintain a strategic distance from Inter Symbol Interference (ISI). QAM have been considered in our dissertation as it supports both an analog and a digital modulation scheme.

The modulation variance considered in our dissertation is 16 to 256 QAM. So it is efficient in checking the performance of BER with respect to multipath fading. Then again the orthogonally of the OFDM depends on the condition that transmitter and collector work with precisely the same recurrence reference. To repay the impact of CFO different $\mathrm{CFO}$ estimations have been considered and good performance has been achieved in case of increasing channel capacity. Temperature variation is also considered with variant channels and the performance is increased in case of more number of channels used.

The following steps showed the process of our work: Step 1: The data distribution according to the probability between 0 to 1 .

Step 2: Different variations of modulations are set to categorized $\mathrm{N}$.

Step 3: Data symbols are generated according to N.

Step 4: Timing jitters are generated.

Step 5: Input $\mathrm{N}$ values.

Step 6: It is transmitted to same timing frame and size.

Step 7: It is added with the prefix input to the receiver side.

Step 8: Different fading channel with multichannel criteria has been applied.

Step 9: Data received at the receiver.

Step 10: BER calculation has been done.

Step 11: Data has been extracted based on the above procedure.

Step 12: The error rate is calculated based on CFO and temperature variants.

\section{Results}

AWGN or white timing jitters affected BER have been shown by circle with dash. Correlated timing jitter affected BER is shown by star with dash. Zero timing jitter affected BER have been shown by plus with dash. Ideal BER performance without interference is shown by $\mathrm{x}$-mark with the dash. The performance of BER based on the AWGN Channel and Rayleigh Fading Channel for $\mathrm{N}=128$ are shown below. Table 1 and Table 2 show the parameter for result comparisons. The results based on the parameters are shown in Figure 1 to Figure 8.

Table 1 Result analysis

\begin{tabular}{lllll}
\hline S. NO & N & L & A & Channel \\
\hline 1 & 128 & 4 & 0.1 & AWGN \\
2 & 128 & 4 & 0.5 & AWGN \\
3 & 128 & 4 & 0.9 & AWGN \\
4 & 128 & 8 & 0.1 & AWGN \\
5 & 128 & 4 & 0.9 & Rayleigh \\
& & & & Fading \\
\hline
\end{tabular}


Mukesh Bhaskar et al.

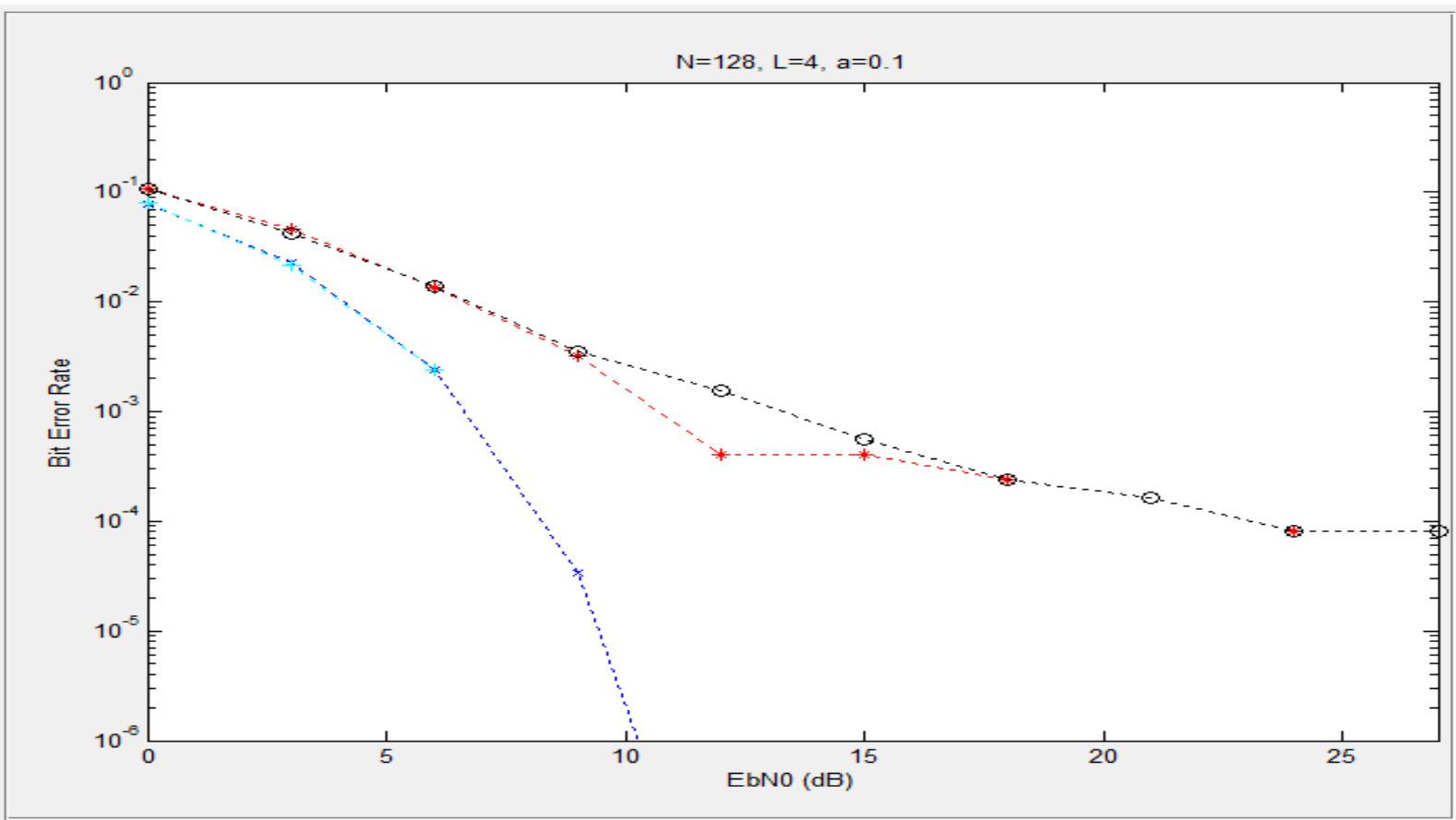

Figure 1 BER Performance under AWGN Channel (parameters $\mathrm{N}=128, \mathrm{~L}=4, \mathrm{a}=0.1$ )

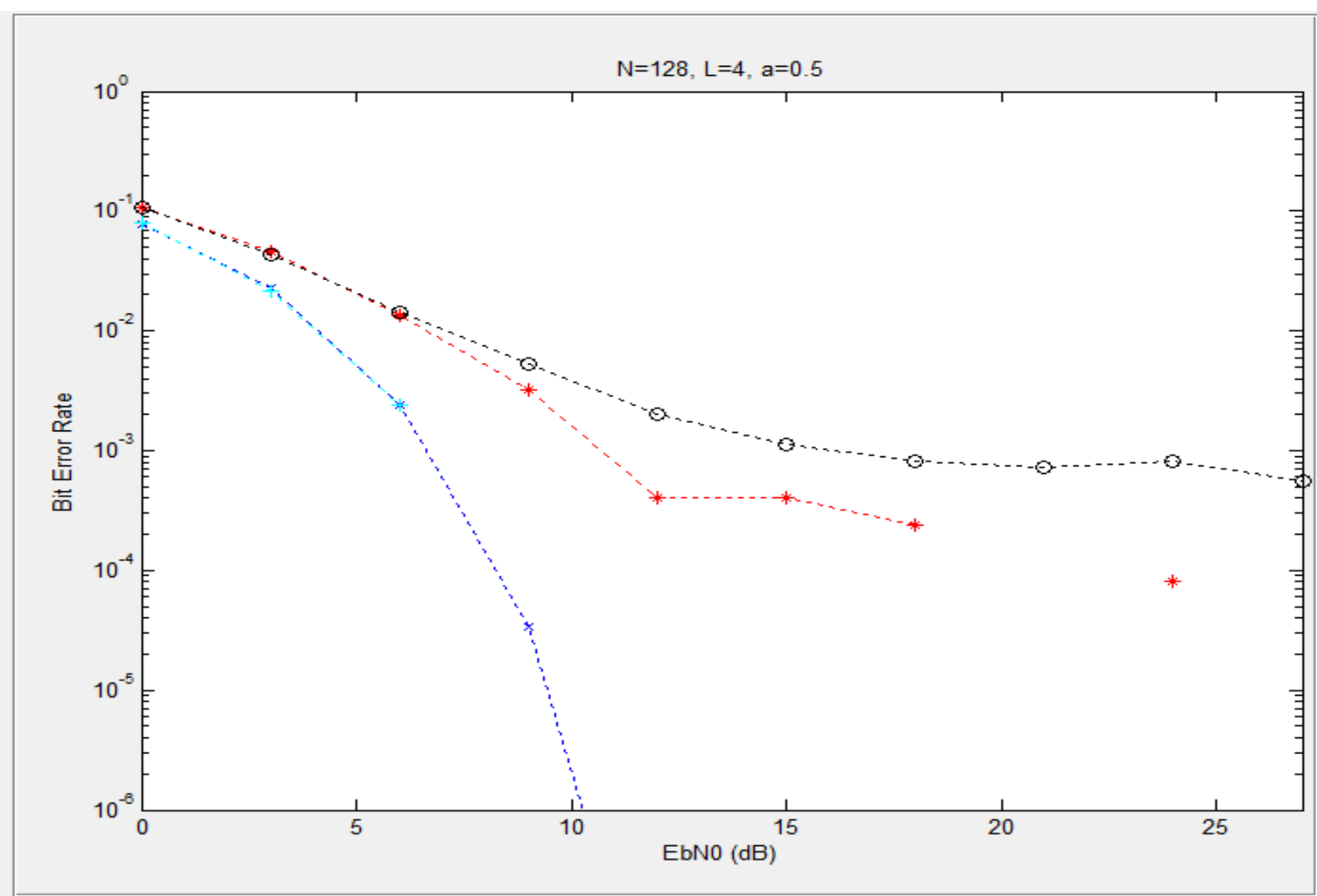

Figure 2 BER Performance under AWGN Channel (parameters $\mathrm{N}=128, \mathrm{~L}=4$, a=0.5) 
International Journal of Advanced Technology and Engineering Exploration, Vol 4(31)

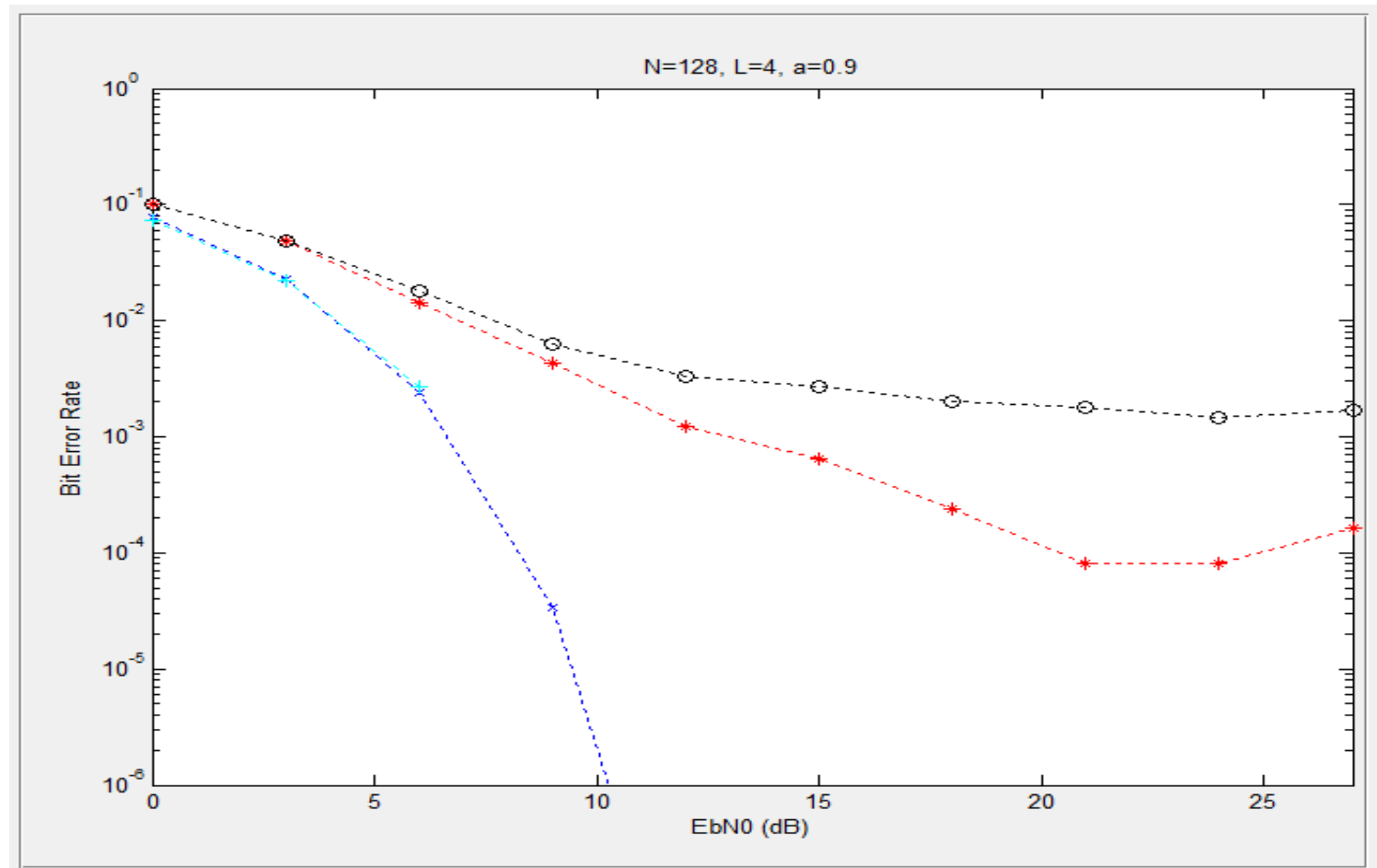

Figure 3 BER Performance under AWGN Channel (parameters $N=128, L=4, a=0.9$ )

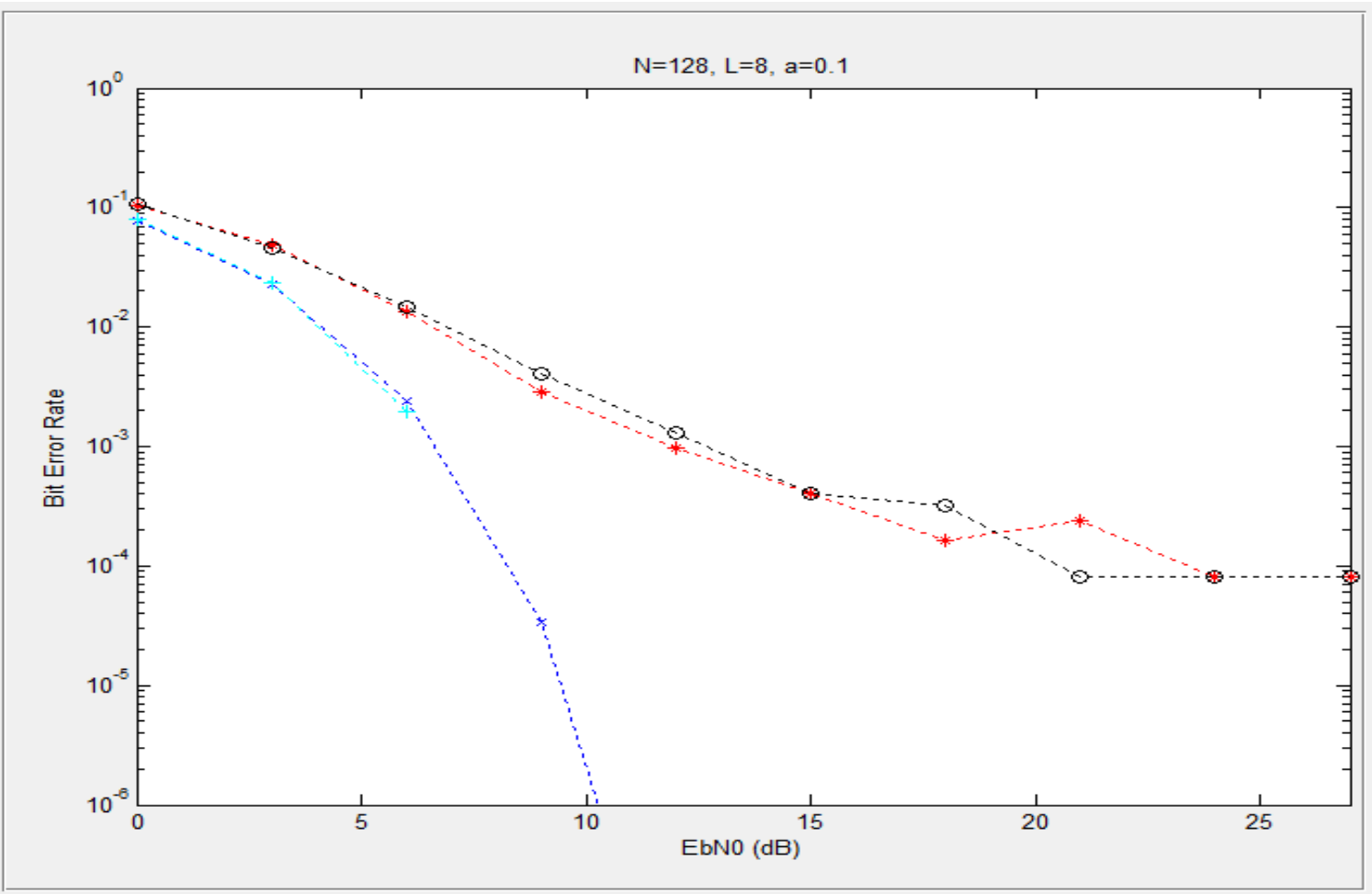

Figure 4 BER Performance under AWGN Channel (parameters $\mathrm{N}=128, \mathrm{~L}=8, \mathrm{a}=0.1$ ) 
Mukesh Bhaskar et al.

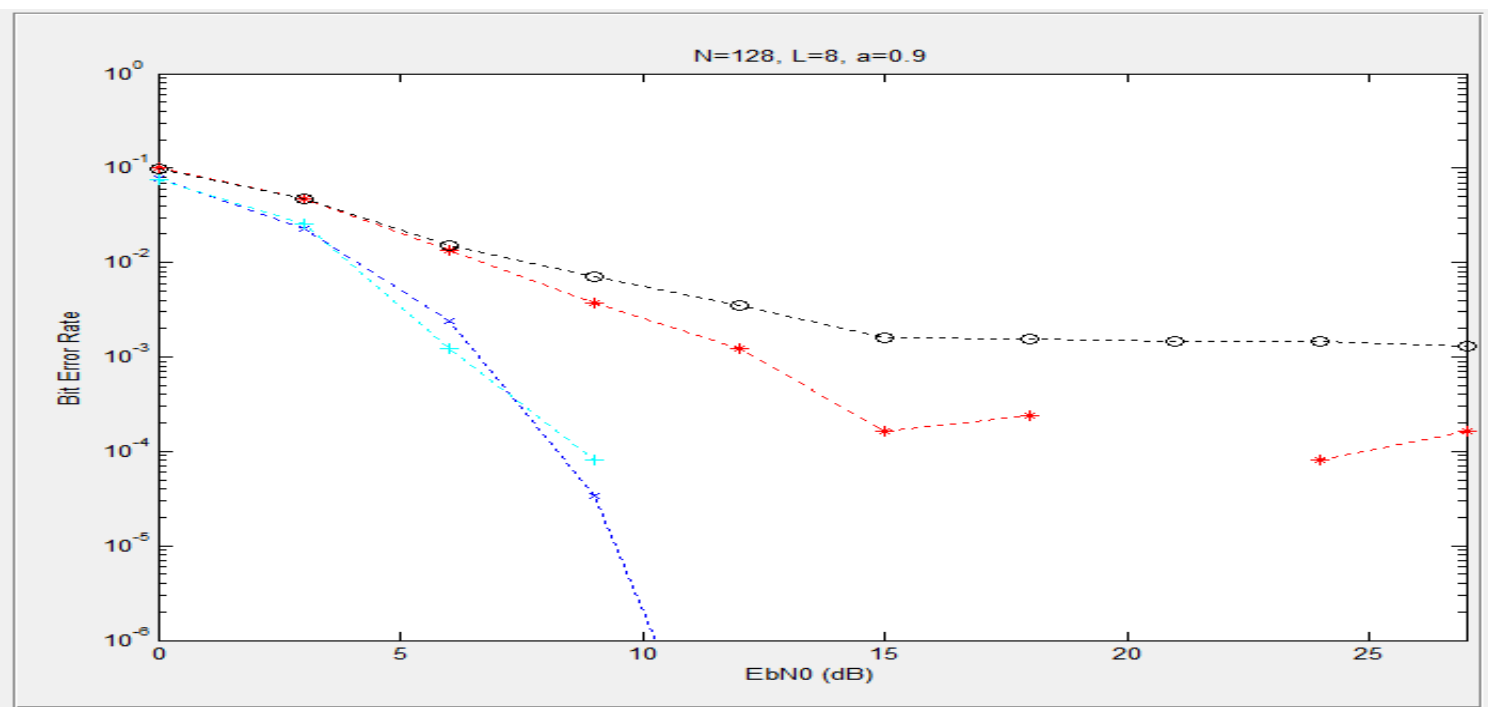

Figure 5 BER Performance under rayleigh fading channel (parameters $\mathrm{N}=128, \mathrm{~L}=8$, a=0.9)

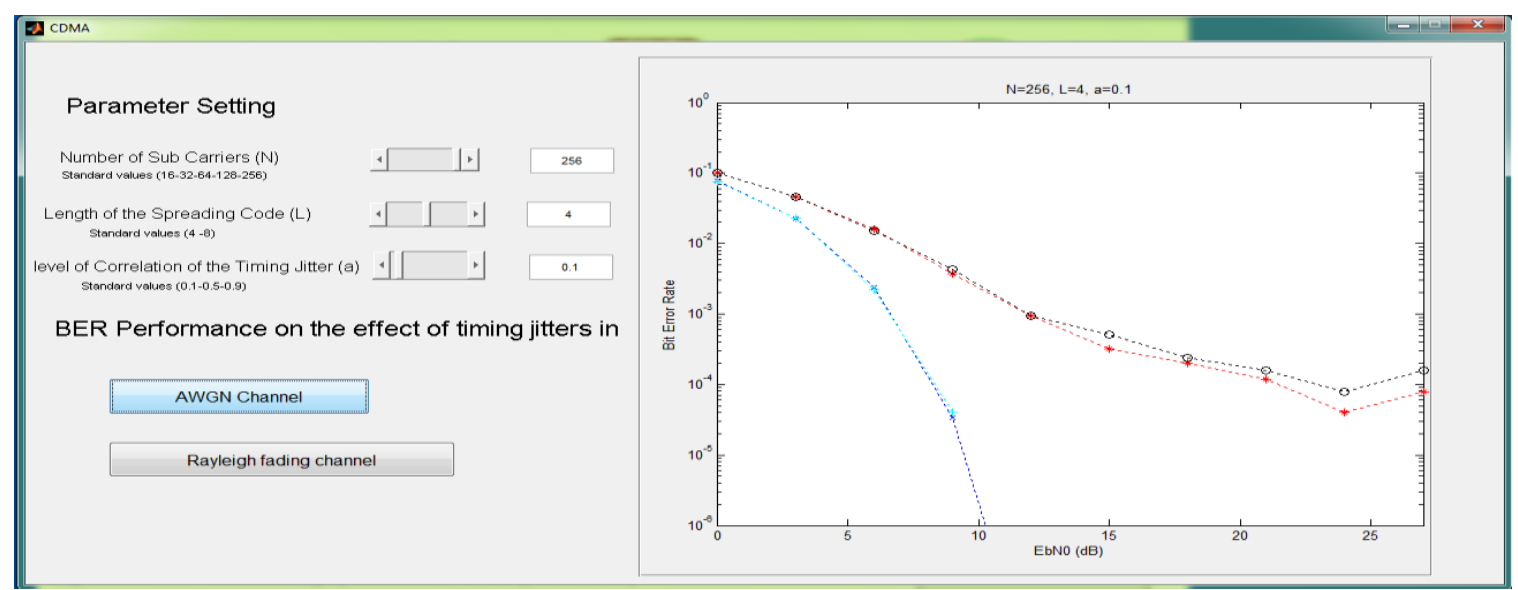

Figure 6 BER Performance under AWGN Channel (parameters N=256, L=4, a=0.1)

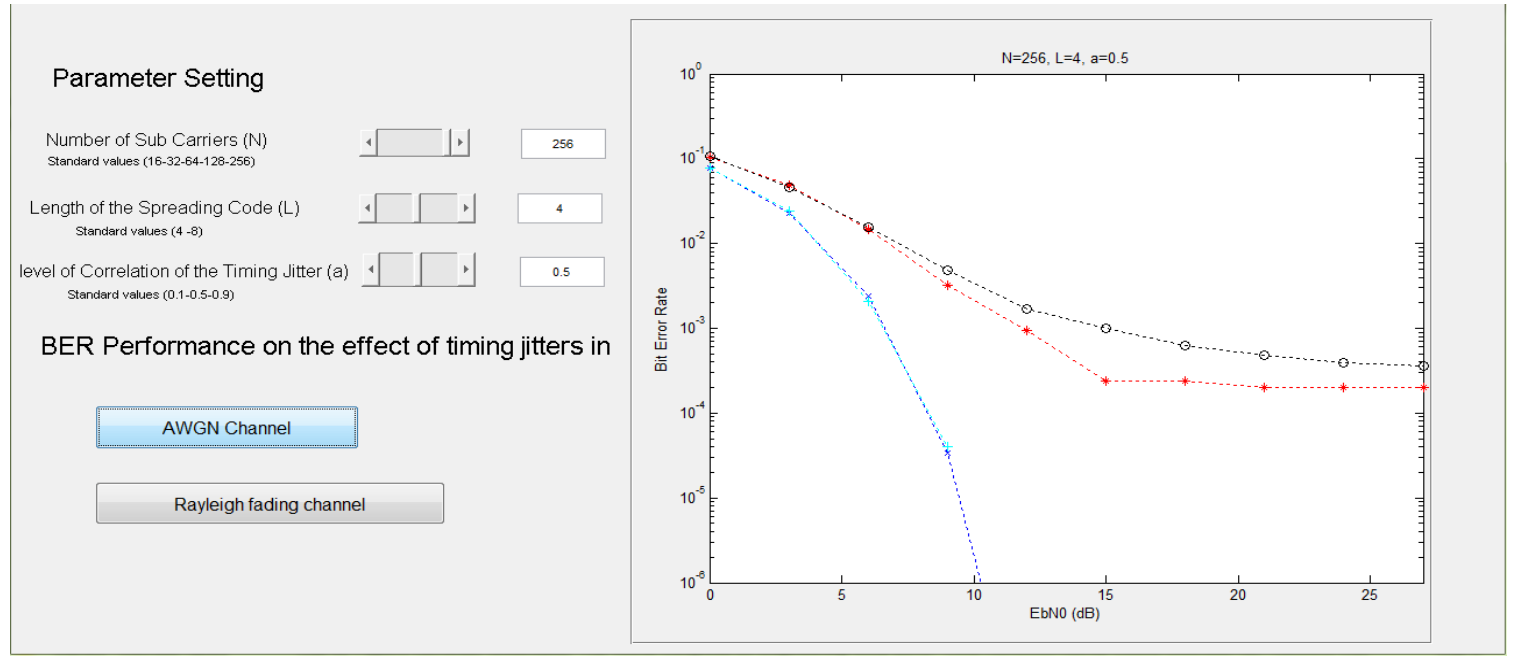

Figure 7 BER Performance under AWGN Channel (parameters N=256, L=4, a=0.5) 


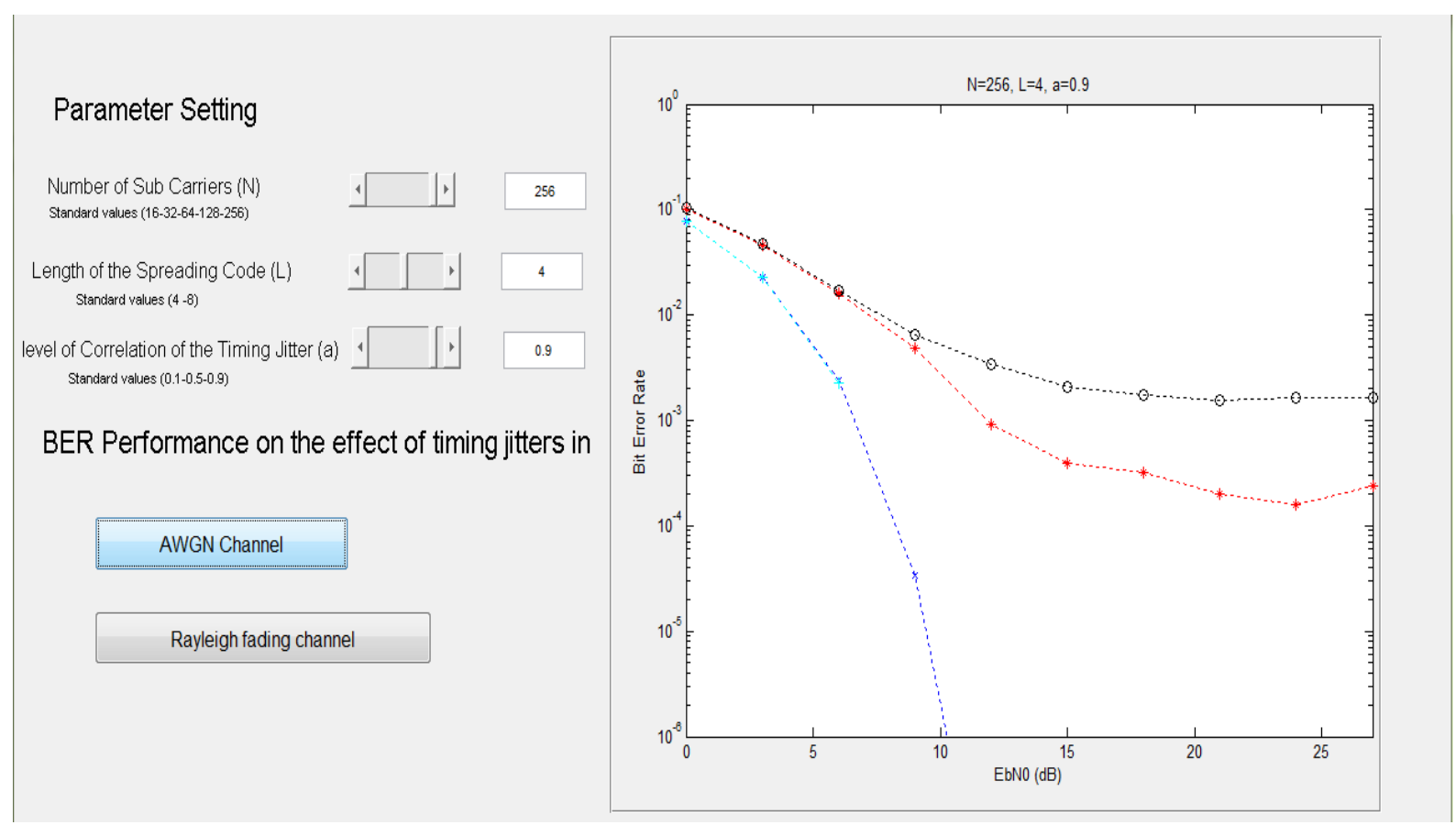

Figure 8 BER Performance under AWGN Channel (parameters $N=256, L=4, a=0.9$ )

Table 2 Result analysis

\begin{tabular}{lllll}
\hline S. NO & N & L & A & Channel \\
\hline 1 & 256 & 4 & 0.1 & AWGN \\
2 & 256 & 4 & 0.5 & AWGN \\
3 & 256 & 4 & 0.9 & AWGN \\
\hline
\end{tabular}

AWGN or white timing jitters affected BER have been shown by circle with dash. Correlated timing jitter affected BER is shown by star with dash. Zero timing jitter affected BER have been shown by plus with dash. Ideal BER performance without interference is shown by $\mathrm{x}$-mark with the dash. The performance of BER based on the AWGN Channel and Rayleigh Fading Channel for $\mathrm{N}=256$ are shown above.

\section{Conclusion}

Our approach suggested an efficient framework on AWGN and Rayleigh channel with the timing jitters and Carrier Frequency Offset control for achieving reducing BER rates. 16, 32, 64, 128 and 256 QAM are used in carrier modulation. The variations in length of timing jitters which is efficient in providing better correlation coefficients. The CFO estimation provides a way of increasing channel capacity with reduced BER rates capability and the impact of $\mathrm{CFO}$ has been discussed with the variation of channels along with the temperature variations.

\section{References}

[1] Frederiksen FB, Prasad R. An overview of OFDM and related techniques towards development of future wireless multimedia communications. In radio and wireless conference 2002 (pp. 19-22). IEEE.

[2] Bingham JA. Multicarrier modulation for data transmission: An idea whose time has come. IEEE Communications Magazine. 1990; 28(5):5-14.

[3] Wang Z, Ma X, Giannakis GB. OFDM or singlecarrier block transmissions? IEEE Transactions on Communications. 2004; 52(3):380-94.

[4] Trivedi S, Raeen MS, Pawar SS. BER analysis of MIMO-OFDM system using BPSK modulation scheme. International Journal of Advanced Computer Research.2012; 2(5):219-26.

[5] Xia P, Giannakis GB. Design and analysis of transmitbeamforming based on limited-rate feedback. IEEE Transactions on Signal Processing. 2006; 54(5):185363.

[6] Paramita S, Singh SS, Mohanta J. Time and frequency synchronization in OFDM system. International Journal of Advanced Computer Research. 2014; 4(3):856-65.

[7] Gupta MV, Tiwari MR. Performance Analysis and Simulation Result of MC-CDMA for AWGN channel and Raleigh based on SNR/BER. International Journal of Advanced Computer Research. 2011; 1(1):13-7.

[8] Rathore M, Tanwar P. Reduction of co-channel interference in transmit/receive diversity (TRD) in MIMO System. International Journal of Advanced Computer Research. 2012; 2(3):13-7.

[9] Gupta P, Singh UP, Richhariya V. Analysis and comparison of the 4-PSK and 8-PSK STTC over 
Mukesh Bhaskar et al.

Rayleigh fading channels for determining performance. International Journal of Advanced Computer Research.2012; 2(5):142-9.

[10] Zhou S, Wang Z, Giannakis GB. Quantifying the power loss when transmit beamforming relies on finite-rate feedback. IEEE Transactions on Wireless Communications. 2005; 4(4):1948-57.

[11] Tarokh V, Seshadri N, Calderbank AR. Space-time codes for high data rate wireless communication: Performance criterion and code construction. IEEE Transactions on Information Theory. 1998; 44(2):74465.

[12] Foschini GJ. Layered space-time architecture for wireless communication in a fading environment when using multi-element antennas. Bell Labs Technical Journal. 1996; 1(2):41-59.

[13] Anitha K, Sujatha BK. FPGA implementation of high throughput digital QPSK modulator using Verilog HDL. International Journal of Advanced Computer Research. 2014; 4(1):217-222.

[14] Foschini GJ, Gans MJ. On limits of wireless communications in a fading environment when using multiple antennas. Wireless Personal Communications. 1998; 6(3):311-35.

[15] Telatar E. Capacity of Multi-antenna Gaussian Channels. Transactions on Emerging Telecommunications Technologies. 1999; 10(6):58595.

[16] Daksh JK, Mohan R, Sharma S. Performance analysis with space-time coding in MIMO-OFDM systems with multiple antennas. International Journal of Advanced Computer Research. 2013; 3(2):126-9.

[17] Choubey R, Mohan R, Sharma S. A survey of BER performance of generalized MC DS-CDMA system. International Journal of Advanced Computer Research. 2013; 3(2):130-3.
[18] Daksh JK, Mohan R, Sharma S. A survey of Performance Analysis in MIMO-OFDM Systems. International Journal of Advanced Computer Research. 2013; 3(2):91-4.

[19] Dubey AK, Khandagre Y, Kushwaha GR, Hemnani K, Tiwari R, Shrivastava N. PAPR reduction in OFDM by using modernize SLM technique. Recent Trends in Wireless and Mobile Networks. 2011:397-405.

[20] Ropokis GA, Rontogiannis AA, Berberidis K. BER performance analysis of cooperative $\mathrm{DaF}$ relay networks and a new optimal DaF strategy. IEEE Transactions on Wireless Communications. 2011; 10(4):1044-9.

[21] Samir M, Shaheen EM, El Wahab AA. Performance analysis of DS-CDMA system using fast adaptive filtering under different jamming techniques. In international conference on engineering and technology 2012 (pp. 1-6). IEEE.

[22] Le ST, Blow KJ, Mezentsev VK, Turitsyn SK. Bit error rate estimation methods for QPSK CO-OFDM transmission. Journal of Lightwave Technology. 2014; 32(17):2951-9.

[23] Zahed MI, Majumder SP. Performance analysis of MC-DS-CDMA in the presence of carrier frequency offset and timing jitter over Rayleigh Fading Channels. In international conference on computer and information technology 2014 (pp. 1-5). IEEE.

[24] Kumar N. BER analysis in Wavelet based SC-FDMA for LTE uplink transmission. In international conference on advanced computing \& communication technologies 2015 (pp. 437-40). IEEE.

[25] Jie Z, Liang LI, Jin LI. Performance analysis of space time block code in MIMO-OFDM systems. In international conference on communication software and networks 2011 (pp. 13-6). IEEE.

[26] Rawat S, Kakde B. A survey on BER performance analysis in AWGN and Rayleigh fading channel. International Journal of Advanced Technology and Engineering Exploration. 2015; 2(7):111-6. 